\title{
Functional imaging in living plants-cell biology meets physiology
}

\author{
George R. Littlejohn ${ }^{1 \dagger}$, Tobias Meckel ${ }^{2 \dagger}$, Markus Schwarzländer ${ }^{3 \dagger}$ and Alex Costa ${ }^{4,5 * t}$ \\ ${ }^{1}$ Division of Plant and Microbial Sciences, School of Biosciences, University of Exeter, Exeter, UK \\ ${ }^{2}$ Membrane Dynamics, Department of Biology, Technische Universität Darmstadt, Darmstadt, Germany \\ ${ }^{3}$ Chemical Signalling, Institute of Crop Science and Resource Conservation, University of Bonn, Bonn, Germany \\ ${ }^{4}$ Department of Biosciences, University of Milan, Milan, Italy \\ ${ }^{5}$ Milan Division, Institute of Biophysics, National Research Council, Milan, Italy \\ *Correspondence: alex.costa@unimi.it \\ these authors have contributed equally to this work.
}

Edited and reviewed by:

Simon Gilroy, University of Wisconsin - Madison, USA

Keywords: in vivo imaging, plants, organelles, cell physiology, dynamics, fluorescent protein sensors, quantitative microscopy

The last quarter of a century has brought major developments in the acquisition of images from plants through improvements in microscopy equipment, software and technique. Likewise, step changes in image analysis tools and the utilization and iterative redesign of fluorescent protein based markers and probes has revolutionized the ability of researchers to ask fundamental questions in cell biology and physiology. This research topic gives a snapshot of the current shape of the field in the plant sciences.

The articles contributed to this research topic are indicative of the work emerging from the plant imaging community and cover, variously, the characterization of individual protein functions; localization and interactions; the use of physiological biosensors; spectroscopic techniques, which utilize autofluorescence of plant tissues and label-free approaches; developmental studies and software engineering. These reflect the broad areas in which imaging is currently being used to functionally dissect plant processes.

A focus in this research topic is the quantitative imaging of fluorescent sensors to explore cell function.

Förster resonance energy transfer (FRET) and how sensitized emission may be used for quantification in vivo imaging is reviewed by Müller et al. (2013) who discuss a set of methods that allows for the analysis of molecular interactions, in the light of recent developments in fluorescence microscopy, which have achieved higher spatial and temporal resolution as well as a much-improved sensitivity. A comprehensive overview of FRET imaging is given with a focus on fluorescent proteins and the procedure and analysis of sensitized emission, which allows for a fast and repetitive monitoring of FRET efficiencies as required for the investigation of dynamic plant cells.

A perspective on the use of genetically encoded fluorescent biosensors (including FRET-based probes) in plants is given by Gjetting et al. (2013). The authors discuss the development of a rapidly growing repertoire of genetically encoded fluorescent sensors and how these developments have been a key driver for functional imaging over the last two decades as well as how new sensors have been adopted by plant biology and future opportunities. Autofluorescence from photosynthetic pigments and secondary metabolites, mounting techniques affecting physiological status, sensor silencing and plant specific compartments, such as the apoplast, are identified as technical burdens that can hamper straightforward sensor usage in plants. Plant-adjusted sensor design, such as the usage of new fluorescent protein variants, and imaging techniques, like fluorescent lifetime imaging (FLIM), are recognized as technical opportunities to advance in vivo sensing in plants. Biological promise comes from bespoke sensing approaches in which the sensor is matched current questions of plant metabolism, physiology and signaling, such as sugar homeostasis, hormone regulation and $\mathrm{pH}$ dynamics of acidic compartments.

The development and properties of $\mathrm{pH}$ probes as one group of the genetically encoded sensors discussed by Gjetting et al. (2013) is given detailed attention and set in biological context by Martinière et al. (2013). Imaging of intracellular $\mathrm{pH}$ dates back to the early efforts to exploit fluorescent proteins as sensors for in vivo physiology. A still growing repertoire of sensor variants for $\mathrm{pH}$ has been extensively applied in plant cells to understand subcellular $\mathrm{pH}$ milieus and proton gradients across membranes. Nevertheless $\mathrm{pH}$ dynamics on a cellular scale are far from understood and potential functional roles of $\mathrm{pH}$ as a central physiological parameter are often elusive. In their perspective article, Martinere et al. shine a spotlight on the opportunities and the persisting technical constraints of $\mathrm{pH}$ imaging in plants. Insights gained from in vivo $\mathrm{pH}$ imaging are discussed with respect to exocytotic function, root apoplast responses to changing environments and growth. The highly dynamic nature of the archipelago of subcellular $\mathrm{pH}$ islands is exemplified for the physiological transition within the endomembrane system between endoplasmic reticulum (ER) and vacuole.

These three review articles set the scene for two original research articles, contributed by Aller et al. (2013) and Xiong et al. (2014) who report the development of new tools for in vivo imaging of glutathione redox status and $\mathrm{Ca}^{2+}$, respectively. Aller et al. (2013) introduce a new member of the redox sensitive GFP family, named roGFP2-iL. roGFP sensors have been extensively used for the in vivo monitoring of glutathione redox potential in both animal and plant cells. The founding members of the family, roGFP1 and roGFP2, have midpoint potentials of -290 and $-280 \mathrm{mV}$ 
respectively, compatible with the monitoring glutathione redox status in plasmatic compartments such as cytosol, mitochondria and peroxisomes. The newly developed roGFP2-iL, which shows a midpoint potential of $-238 \mathrm{mV}$, now enables measurement of glutathione redox status under more oxidizing circumstances, such as in genetic backgrounds with impaired thiol redox maintenance (here exemplified for the glutathione deficient Arabidopsis rmll mutant) or in the ER, where both roGFP1 and roGFP2 are completely oxidized. This makes a powerful enhancement of the toolset of glutathione redox sensors and shifts the redox frontier allowing to explore new biology not only in plants.

Addressing another hub of regulation, Xiong et al. (2014) introduce a Bioluminescence Resonance Energy Transfer (BRET) sensor for $\mathrm{Ca}^{2+}$ in Arabidopsis. The BRET sensor "re-unites" old partners from the jellyfish Aequoria victoria-aequorin and GFP, enabling imaging of $\mathrm{Ca}^{2+}$ dynamics in entire seedlings and mature leaves of Arabidopsis without the necessity of illumination, as required for other popular $\mathrm{Ca}^{2+}$ sensor classes, such as the Yellow Cameleons, the GCaMPs and Case12. Instead the GFP-aequorin (G5A) sensor harnesses the photons emitted by the aequorin-coelenterazine complex upon binding of $\mathrm{Ca}^{2+}$ to excite the adjacent GFP, via BRET, the fluorescence of which can be detected with a cooled charge-coupled device camera (CCD). This approach allows for increased sensitivity as compared to standard aequorin-based $\mathrm{Ca}^{2+}$ sensing and enabled the authors to monitor long-distance $\mathrm{Ca}^{2+}$ waves propagating throughout the entire plant body after salt stress treatment applied to the root. This new $\mathrm{Ca}^{2+}$ imaging approach will complement other recently developed tools for the in vivo analysis of this central second messenger.

Instead of applying fluorescent dyes or introducing recombinant sensor proteins, the same autofluorescence by endogenous plant compounds highlighted as a burden for quantitative imaging by Gjetting et al. (2013) may be actively exploited to provide valuable physiological insight. New spectroscopic techniques for label-free imaging to investigate plant physiology are presented by Peter et al. (2014) and Conejero et al. (2014).

Peter et al. (2014) use spectro-microscopy and Statistical Analysis of Room Temperature Emission Spectra (SART) to characterize in vivo function of photosystems PSI and PSII in chloroplasts. This non-invasive technique exploits the natural light absorbance properties of chloroplasts and has the ability to deliver photosynthetic parameters for single chloroplasts at normal growth conditions. As this technique requires only moderate modification of a confocal microscope, it may be readily implemented by well-resourced laboratories.

Conejero et al. (2014) present a method combining spectral analysis with linear unmixing to facilitate histolocalization of phenolics in coffee leaves. Their protocol involves twophoton excitation, spectral characterization of pure chemicals and advanced linear unmixing. Conejero et al. (2014) are able to follow the amount and distribution of key phenolic compounds throughout the development of leaves of various Coffea species. By obviating the need for any staining, truly noninvasive histochemical analysis based on quantitative imaging is achieved.
Label-free SRS microscopy is used by Littlejohn et al. (2014) to delimit the negative-space in plant leaves in their paper updating the use of perfluorocarbon mounting media in plants leaves. Functional imaging in intact, living leaves as the main organ of photosynthesis, is often particularly desirable. Much work has been performed on leaf epidermis, while high quality imaging of the mesophyll or vascular bundle cells can prove challenging, due to the optical complexity of the tissue with multiple stacked cell layers and air spaces. Littlejohn et al. (2014) empirically assess the usage of perfluorocarbons, as non-aqueous and non-toxic mounting media for in vivo microscopy. A systematic comparison of yet untested perfluorocarbons with four stateof-the-art microscopy techniques pinpoints strong advantages for image quality from the use of perfluoroperhydrophenanthrene. This methodological advance goes far beyond producing "prettier images" and opens a new window for the quantitative in vivo study of a defining plant tissue. A particular benefit may be anticipated for ratiometric sensing approaches of physiology where increasing noise and chromatic aberrations in deeper tissue layers can hamper accurate quantitation.

Sattarzadeh et al. (2013) provide an example of how the use of confocal and spinning disc microscopy and in vivo imaging facilitates the definition of subcellular localization of proteins through the generation of chimeric fusion constructs between a fluorescent protein (e.g. GFP, YFP, RFP) and the full protein of interest or a functional domain. The authors identify conserved 42 amino-acid PAL domains present in 12 of the 13 Arabidopsis class XI myosin isoforms. YFP translational fusions for 11 different myosin PAL sub-domains allowed determination of their subcellular localization at Golgi, mitochondria, nuclear envelope, the plasma membrane and unidentified vesicles. Interestingly, the simultaneous expression of three PAL sub-domains resulted minimal or negligible movement of Golgi and mitochondria, allowing the authors not only to demonstrate that different YFPPAL sub-domains localize to different subcellular compartments, but also that their overexpression can interfere with the mobility of the marked compartments in the cell. This work illustrates the elegance of in vivo imaging in exploring dynamic cell biological processes.

To extract the relevant information out of the highly complex dataset that is an image quantitative evaluation is essential, but far from trivial. In the field of plant cell morphology, cell shapes are irregular and highly variable, which requires the use of quantitative techniques to accurately define shape and provide well-defined phenotypic descriptions. In their review Ivakov and Persson (2013) present the current state of knowledge on cell shape formation in plants, focusing on the use of new quantitative tools and algorithms required to quantify and compare cell shapes in $2 \mathrm{D}$ and $3 \mathrm{D}$ obtained from microscope images.

This research topic reflects the breadth of approaches developed, adjusted and followed by the plant community in terms of sample preparation and image acquisition and analysis. A major driver behind the recent progress on the burning questions in plants sciences have been technological advances in imaging. Yet the field is far from maturity and progresses quickly with the promise of keeping delivering fundamental new insights in the years to come. 


\section{REFERENCES}

Aller, I., Rouhier, N., and Meyer, A. J. (2013). Development of roGFP2-derived redox probes for measurement of the glutathione redox potential in the cytosol of severely glutathione-deficient rml1 seedlings. Front. Plant Sci. 4:506. doi: 10.3389/fpls.2013.00506

Conejero, G., Noirot, M., Talamond, P., and Verdeil, J.-L. (2014). Spectral analysis combined with advanced linear unmixing allows for histolocalization of phenolics in leaves of coffee trees. Front. Plant Sci. 5:39. doi: 10.3389/fpls.2014. 00039

Gjetting, S. K., Schulz, A., and Fuglsang, A. T. (2013). Perspectives for using genetically encoded fluorescent biosensors in plants. Front. Plant Sci. 4:234. doi: 10.3389/fpls.2013.00234

Ivakov, A., and Persson, S. (2013). Plant cell shape: modulators and measurements. Front. Plant Sci. 4:39. doi: 10.3389/fpls.2013.00439

Littlejohn, G. R., Mansfield, J. C., Christmas, J. T., Witterick, E., Fricker, M. D., Grant, M. R., et al. (2014). An update: improvements in imaging perfluorocarbon-mounted plant leaves with implications for studies of plant pathology, physiology, development and cell biology. Front. Plant Sci. 5:140. doi: 10.3389/fpls.2014.00140

Martinière, A., Desbrosses, G., Sentenac, H., and Paris, N. (2013). Development and properties of genetically encoded $\mathrm{pH}$ sensors in plants. Front. Plant Sci. 4:523. doi: $10.3389 /$ fpls.2013.00523

Müller, S. M., Galliardt, H., Schneider, J., Barisas, B. G., and Seidel, T. (2013). Quantification of Förster resonance energy transfer by monitoring sensitized emission in living plant cells. Front. Plant Sci. 4:413. doi: 10.3389/fpls.2013.00413

Peter, S. B., Zell, M. B., Blum, C., Stuhl, A., Elgass, K., Sackrow, M., et al. (2014). Photosynthesis in a different light: spectro-microscopy for in vivo characterization of chloroplasts. Front. Plant Sci. 5:292. doi: 10.3389/fpls.2014. 00292

Sattarzadeh, A., Schmelzer, E., and Hanson, M. R. (2013). Arabidopsis myosin $\mathrm{XI}$ sub-domains homologous to the yeast myo2p organelle inheritance subdomain target subcellular structures in plant cells. Front. Plant Sci. 4:407. doi: 10.3389/fpls.2013.00407

Xiong, T. C., Ronzier, E., Sanchez, F., Corratgé-Faillie, C., Mazars, C., and Thibaud, J.-B. (2014). Imaging long distance propagating calcium signals in intact plant leaves with the BRET-based GFP-aequorin reporter. Front. Plant Sci. 5:43. doi: $10.3389 /$ fpls. 2014.00043

Conflict of Interest Statement: The authors declare that the research was conducted in the absence of any commercial or financial relationships that could be construed as a potential conflict of interest.

Received: 19 November 2014; accepted: 04 December 2014; published online: 19 December 2014.

Citation: Littlejohn GR, Meckel T, Schwarzländer M and Costa A (2014) Functional imaging in living plants—cell biology meets physiology. Front. Plant Sci. 5:740. doi: $10.3389 / \mathrm{fpls} .2014 .00740$

This article was submitted to Plant Cell Biology, a section of the journal Frontiers in Plant Science.

Copyright (C) 2014 Littlejohn, Meckel, Schwarzländer and Costa. This is an openaccess article distributed under the terms of the Creative Commons Attribution License (CC BY). The use, distribution or reproduction in other forums is permitted, provided the original author(s) or licensor are credited and that the original publication in this journal is cited, in accordance with accepted academic practice. No use, distribution or reproduction is permitted which does not comply with these terms. 